\title{
Adenoviral targeting of malignant melanoma for fluorescence- guided surgery prevents recurrence in orthotopic nude-mouse models
}

\author{
Shuya Yano ${ }^{1,2,3}$, Kiyoto Takehara ${ }^{1,2,3}$, Hiroyuki Kishimoto ${ }^{3}$, Yasuo Urata4, Shunsuke \\ Kagawa $^{3}$, Michael Bouvet ${ }^{2}$, Toshiyoshi Fujiwara ${ }^{3}$, Robert M. Hoffman ${ }^{1,2}$ \\ ${ }^{1}$ AntiCancer, Inc., San Diego, CA, USA \\ ${ }^{2}$ Department of Surgery, University of California San Diego, La Jolla, CA, USA \\ ${ }^{3}$ Department of Gastroenterological Surgery, Okayama University, Graduate School of Medicine, Dentistry and Pharmaceutical \\ Sciences, Okayama, Japan \\ ${ }^{4}$ Oncolys BioPharm Inc., Tokyo, Japan \\ Correspondence to: Robert M. Hoffman, e-mail: all@anticancer.com \\ Toshiyoshi Fujiwara, e-mail: toshi_f@md.okayama-u.ac.jp \\ Keywords: melanoma, nude mice, fluorescence-guided surgery (FGS), adenovirus, OBP-401 \\ Abbreviations: GFP, green fluorescent protein; RFP, red fluorescent protein; FGS, fluorescence-guided surgery; BLS, bright- \\ light surgery \\ Received: September 17, $2015 \quad$ Accepted: November 27, $2015 \quad$ Published: December 18, 2015
}

\section{ABSTRACT}

\begin{abstract}
Malignant melanoma requires precise resection in order to avoid metastatic recurrence. We report here that the telomerase-dependent, green fluorescent protein (GFP)-containing adenovirus OBP-401 could label malignant melanoma with GFP in situ in orthotopic mouse models. OBP-401-based fluorescence-guided surgery (FGS) resulted in the complete resection of malignant melanoma in the orthotopic models, where conventional bright-light surgery (BLS) could not. High-dose administration of OBP-401 enabled FGS without residual cancer cells or recurrence, due to its dual effect of cancer-cell labeling with GFP and killing.
\end{abstract}

\section{INTRODUCTION}

Despite recent advances, the current therapeutic approaches in melanoma are not satisfactory and intensive research in this area is still required [1].

Cancer surgery requires precise identification of tumor margins. Green fluorescent protein (GFP) fluorescence can intensely illuminate even single cells in vivo $[2,3]$. Introducing and selectively activating the GFP gene in tumors in vivo was made possible by the development of OBP-401, a telomerase-dependent, replication-competent adenovirus expressing GFP. In an intraperitoneal nude mouse model of disseminated human colon cancer, fluorescence-guided surgery (FGS) enabled resection of tumor nodules labeled with GFP by OBP-401 [4]. Recurrent tumor nodules brightly expressed GFP, indicating that initial OBP-401-GFP labeling of peritoneal disease was genetically stable [5].

Glioblastoma multiforme (GBM) is one of the most invasive of cancers and is not totally resectable using standard bright-light surgery (BLS) or current FGS strategies. We previously developed a curative strategy for FGS of GBM using high-dose OBP-401 to selectively label GBM with GFP. OBP-401-based FGS enabled curative resection of GBM without recurrence for at least 150 days, compared to less than 30 days with BLS [6].

OBP-401-based FGS resulted in superior resection of soft-tissue sarcoma (STS) in an orthotopic nude mouse model, compared to BLS. High-dose OBP-401 enabled FGS without residual sarcoma cells or local or metastatic recurrence, due to its dual effect of cancer-cell labeling with GFP and killing. High-dose OBP-401 based-FGS improved disease-free survival as well as preserved muscle function compared with BLS [7].

OBP-401 was used to label the cancer cells of a pancreatic cancer patient-derived orthotopic xenograft (PDOX) nude mouse model. The PDOX was previously grown in a red fluorescent protein (RFP) transgenic mouse that stably labeled the PDOX stroma cells bright red. The color-coded PDOX model enabled FGS to completely resect the pancreatic tumors including stroma. Dualcolored FGS significantly prevented local recurrence, 
which bright-light surgery or single-color FGS could not. FGS, with color-coded cancer and stroma cells has important potential for improving the outcome of recalcitrant-cancer surgery [8].

OBP-401-FGS of tumors in the lung enabled complete lung tumor resection with no residual fluorescent tumor [9].

OBP-401 tumor illumination enabled effective FGS of an orthotopic mouse model of human osteosarcoma model as well as eradication of residual osteosarcoma cells after BLS. OBP-401-FGS significantly inhibited local recurrence and lung metastasis after surgery thereby prolonging survival [10].

In the present report, we demonstrate that OBP-401

GFP labeling in situ enables precise FGS of malignant melanoma without recurrence.

\section{RESULTS AND DISCUSSION}

\section{OBP-401 targets malignant melanoma cell lines with GFP in vitro}

Time-course imaging demonstrated that OBP-401 labeled RFP-expressing Mel526, FEMX1, LOMVIXI, MV3 malignant melanoma cells with GFP (Figure 1A). GFP fluorescence, after OBP-401 targeting of Mel526, FEMX1, LOMVIXI, MV3 cells, became stronger each day from day 2 to day 7 (Figure 1A, 1B).

\section{Orthotopic malignant melanoma model}

An orthotopic malignant melanoma tumor model was established with FEMX1-RFP cells implanted in the femoral skin where FEMX1-RFP cells produced a nodular tumor (Supplementary Figure S1A, S1B). The orthotopicallygrowing malignant melanoma cells invaded the quadriceps femoris muscle and metastasized to lymph nodes similar to the clinical course of melanoma (Supplementary Figure S1C). Tumor growth was visualized by RFP fluorescence (Supplementary Figure S1C).

\section{Bright-light surgery results in residual melanoma cells in the orthotopic model}

We performed bright-light surgery (BLS) on the orthotopic malignant melanoma model (Figure 2A, Supplementary Figure S2A). Due to inability to clearly visualize the tumor margins, extensive RFP-expressing malignant melanoma cells remained after BLS (Figure 2A, 2D).

\section{OBP-401-based fluorescence-guided surgery (OBP-401-FGS) of orthotopic melanoma using a stationary imaging system}

The orthotopic tumor growing in the femoral skin $\left(100 \mathrm{~mm}^{3}\right.$, diameter; $\left.6 \mathrm{~mm}\right)$ was resected 3 days after i.t. injection of OBP-401 $\left(1 \times 10^{8} \mathrm{PFU}\right)$ (Figure 2B,
Supplementary Figure S2B). OBP-401 conferred GFP fluorescence of the orthotopic melanoma which was sufficiently bright to perform complete resection using the OV100 whole body imaging system (Figure 2B). Tumor imaging showed that OBP-401 GFP labeling co-localized with tumor RFP fluorescence (Figure 2B, 2C). OBP401-GFP-targeted FGS resulted in no detectable residual melanoma cells (Figure 2B, 2D).

\section{OBP-401-FGS of malignant melanoma using a hand-held portable fluorescence imaging system}

OBP-401-GFP targeting enabled the use of the Dino-Lite hand-held portable fluorescence imager [11] for FGS (Figure 3). OBP-401-GFP labeling for four days made the tumor margin much clearer than under brightlight (Figure 3A). Using the Dino-Lite, the tumor margin was clearly visualized enabling complete resection of the malignant melanoma (Figure 4B, Supplementary Movie S1). Fluorescence imaging showed that there were no residual cancer cells after OBP-401 FGS of malignant melanoma with the Dino-Lite (Figures 3, 4).

\section{OBP-401-FGS targets residual melanoma cells in the orthotopic model after BLS and enables complete resection}

OBP-401 was intratumorally injected 3 days before BLS of FEMX1-RFP melanoma growing in the femoral skin (Supplementary Figure 3). OBP-401 enabled detection of the residual cancer cells at the single cell level in the surgical bed after BLS using a whole body imaging system (OV100) (Figure 4A). After OBP-401-FGS, there were no detectable residual cancer cells (Figure 4B, 4C). OBP-401 delineated the precise margins between cancer and normal tissue. OBP-401 enabled determination whether there were residual cancer cells at the singlecell level after FGS using confocal microscopy (FV1000) (Figure 4D, 4E).

\section{High-dose OBP-401 kills melanoma cells growing orthotopically in vivo, thereby enabling minimal surgery}

High-dose OBP-401 targeted and killed Mel526RFP, FEMX1-RFP, LOMVIXI-RFP, MV3-RFP cells in a dose-dependent manner in vitro (Figure 5). Therefore, we determined whether high-dose OBP-401 $\left(2 \times 10^{8}\right.$ PFU)FGS enabled minimally-invasive surgery of melanoma compared with BLS or low-dose FGS. Highdose OBP-401 injected i.t. significantly reduced the size of tumors compared with untreated control tumors or low-dose OBP-401 (Figure 6A, 6B). The surgical area necessary for high-dose OBP-401 FGS was smaller than that for BLS or low-dose OBP-401 FGS (Figure 6C, 6D). The weight of tumor resected by high-dose OBP-401 FGS 
A
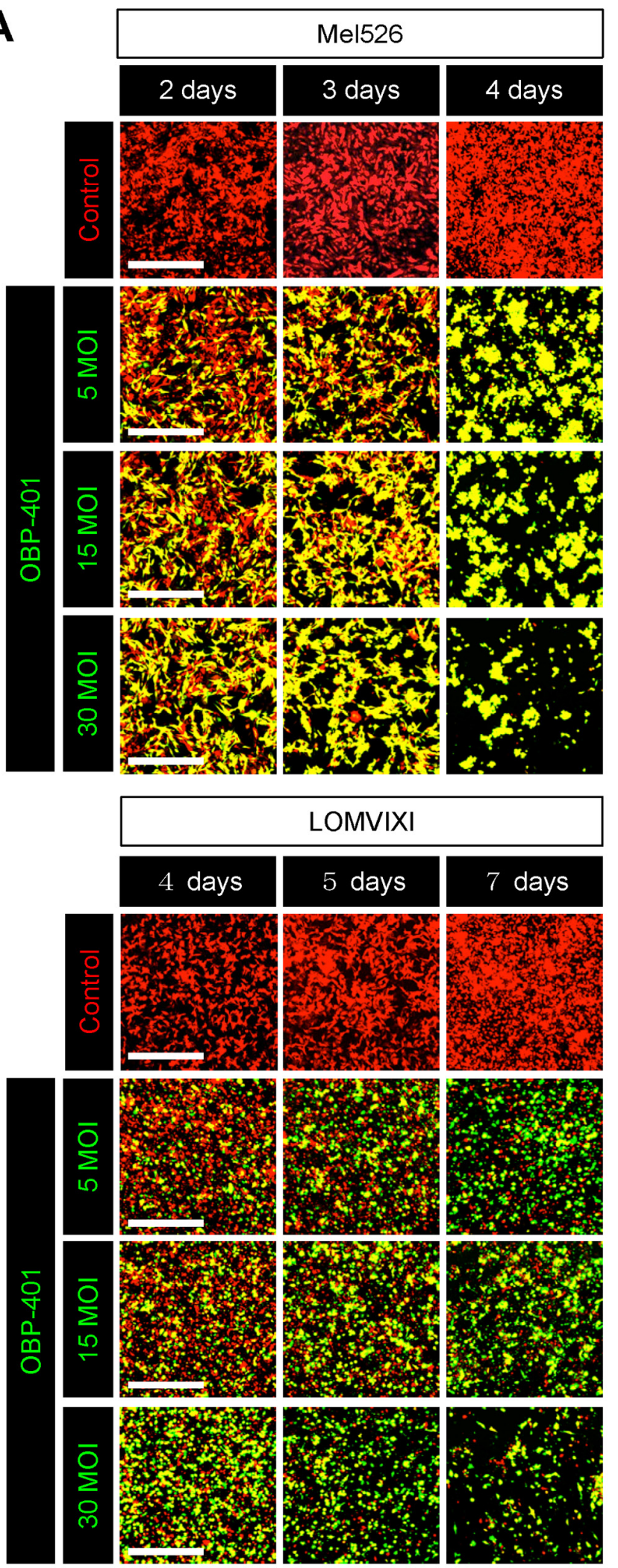
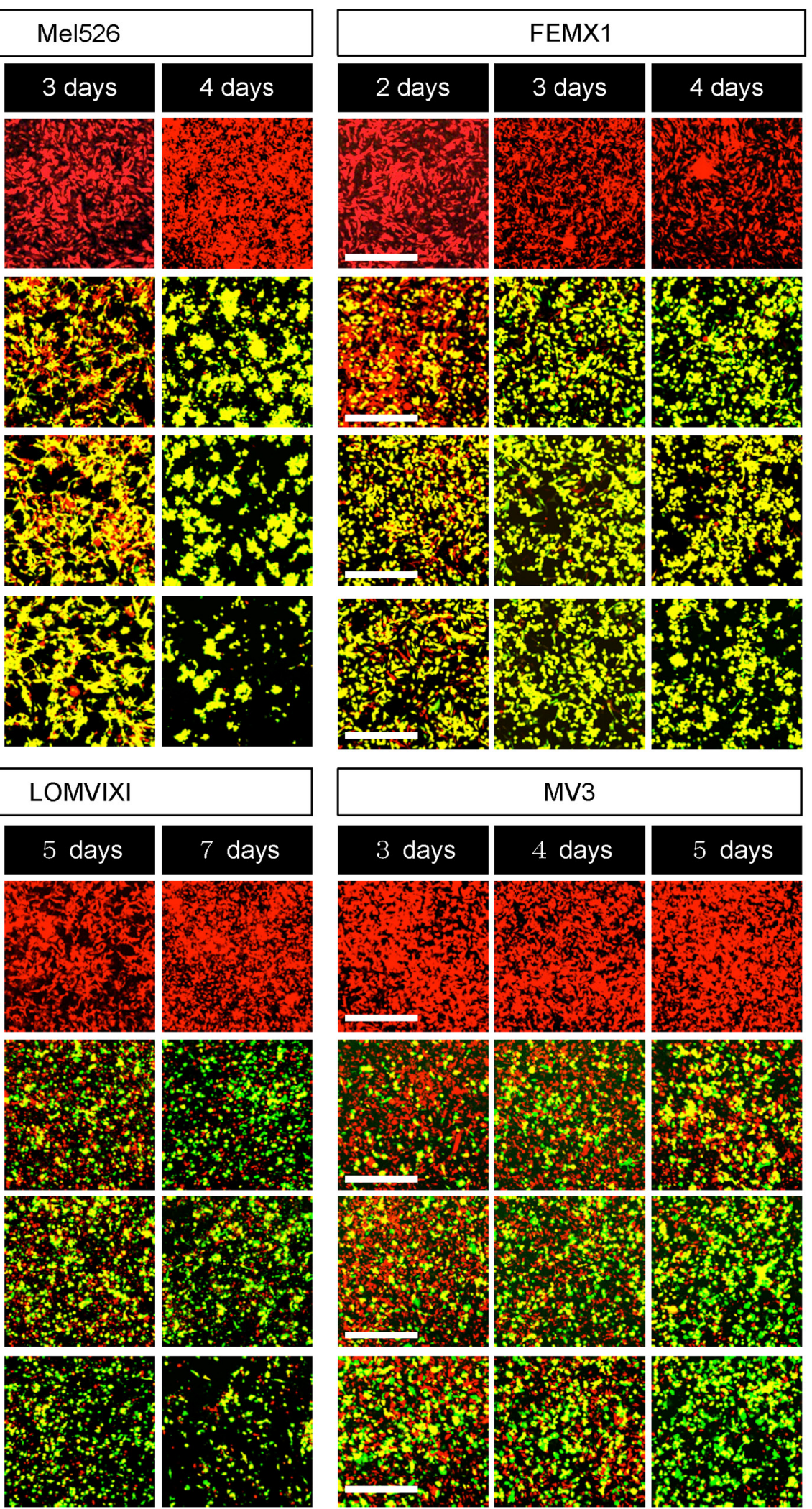

Figure 1. OBP-401 targets human malignant melanoma cell lines with GFP in vitro. RFP-expressing human malignant melanoma cell lines, Mel526, FEMX1, LOMVIXI, MV3 were seeded on 6 well plates $\left(1 \times 10^{5}\right.$ cells well). OBP-401 was added at the indicated multiple of infection (MOI) 24 hours after cell seeding. Images were acquired with an FV1000 confocal laser scanning microscope (Olympus). A. Timecourse images of Mel526, FEMX1, LOMVIXI, MV3 cells 2, 3, and 4 days after infection with OBP-401, at indicated MOIs.

(Continued) 
B

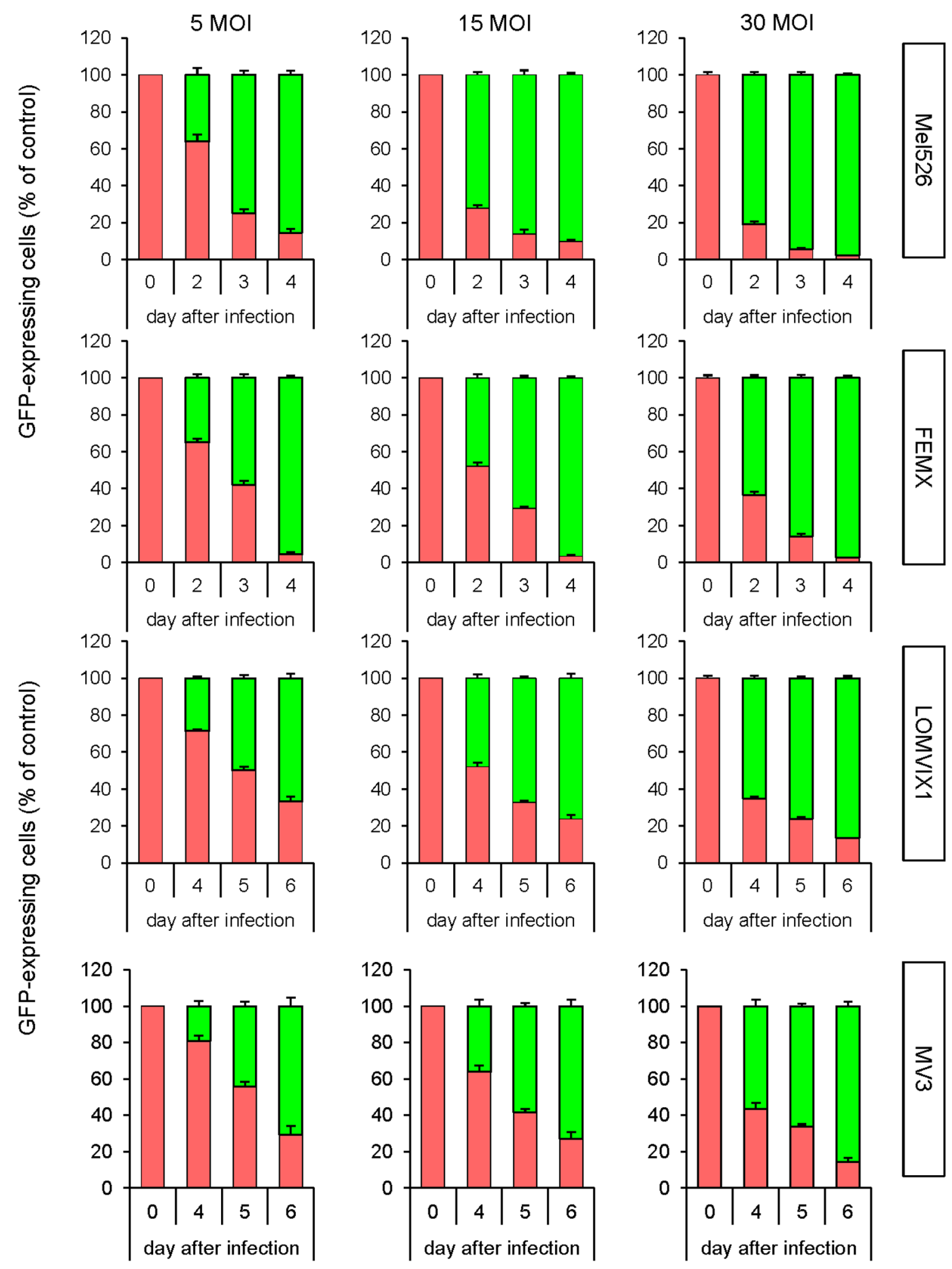

Figure 1 (Continued): B. Histograms show the frequency of GFP-expressing Mel526, FEMX1, LOMVIXI, MV3 cells at indicated days after infection with OBP-401. Data are shown as average \pm SD. $N=5$. Scale bars $=500 \mu \mathrm{m}$. 
A
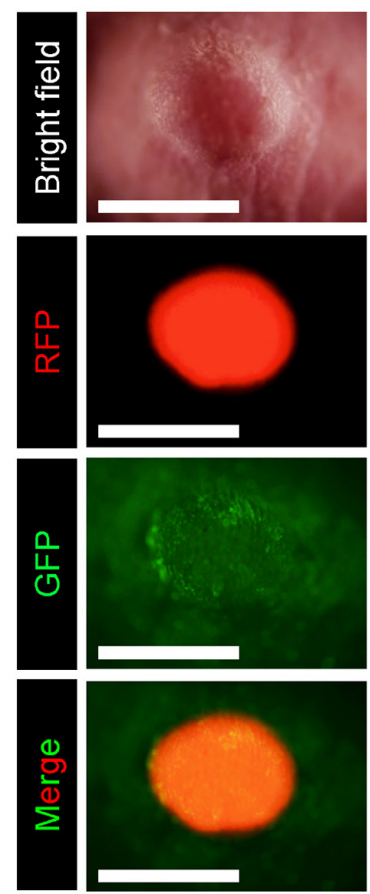

B
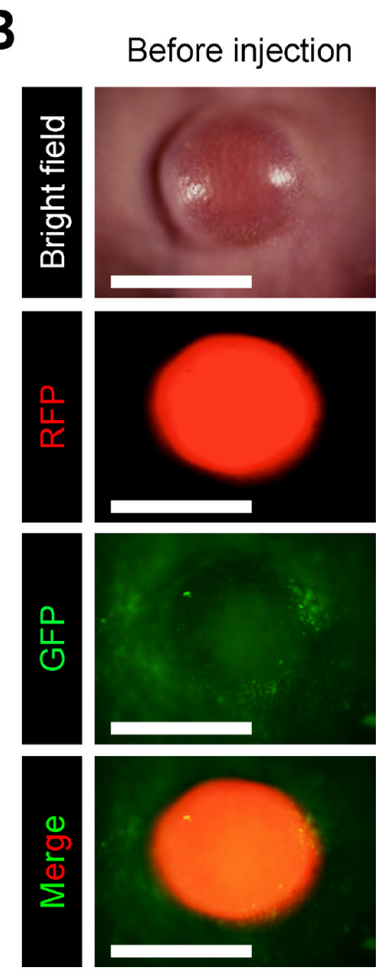

Day 3
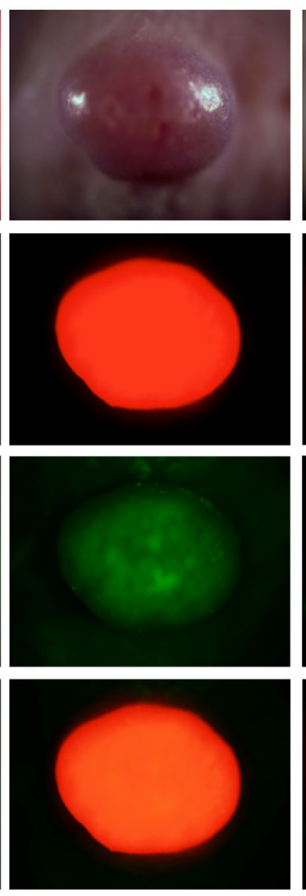

3 days after injection
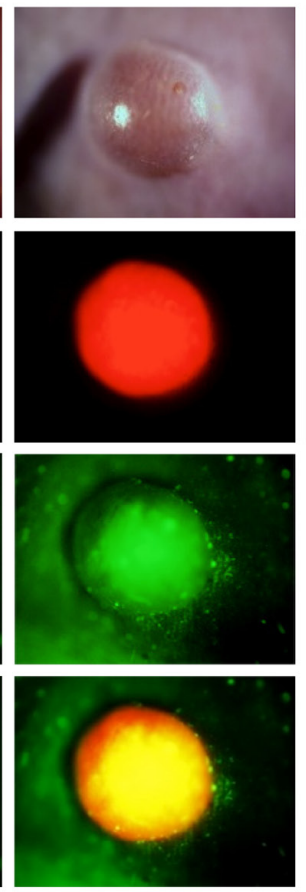

Before BLS
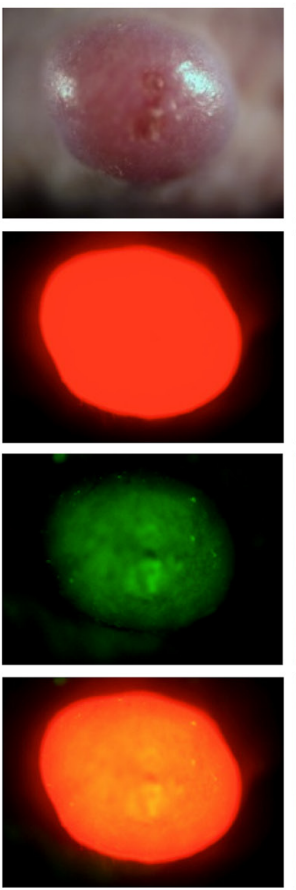

Before FGS
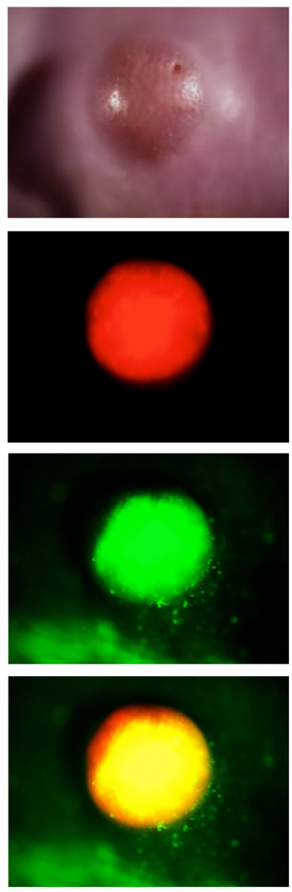

After BLS
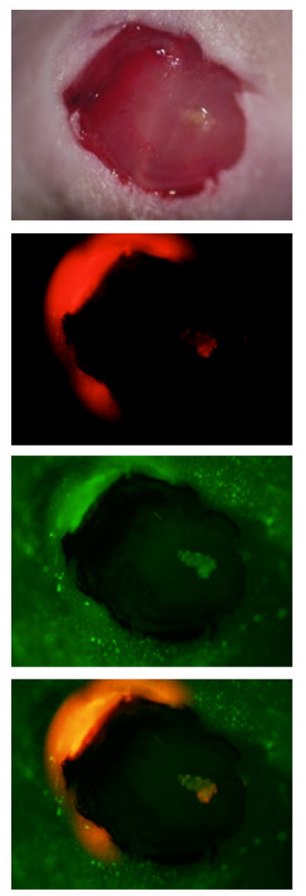

After FGS
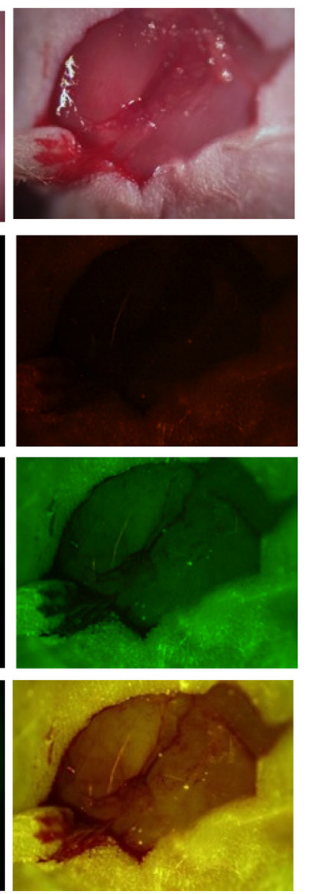

Figure 2. Comparison of OBP-401-targeted fluorescence-guided surgery with bright-light surgery for orthotopic malignant melanoma using a stationary imaging system. RFP-expressing FEMX1 cells $\left(5 \times 10^{\circ}\right)$ in $20 \mu 1$ Matrigel (BD) were inoculated in the femoral skin of nude mice (5 weeks old). OBP-401 $\left(1 \times 10^{8} \mathrm{PFU}\right)$ was injected intratumorally when tumors reached approximately $100 \mathrm{~mm}^{3}$ (6 mm diameter). A. Representative whole-tumor images of mock-infected REP-expressing orthotopic melanoma before and after bright-light surgery (BLS). B. Representative whole-tumor images of OBP-401-GFP labeled, RFP-expressing orthotopic malignant melanoma before and after OBP-401-based fluorescence-guided surgery (OBP-401 FGS).

(Continued) 


\section{C}
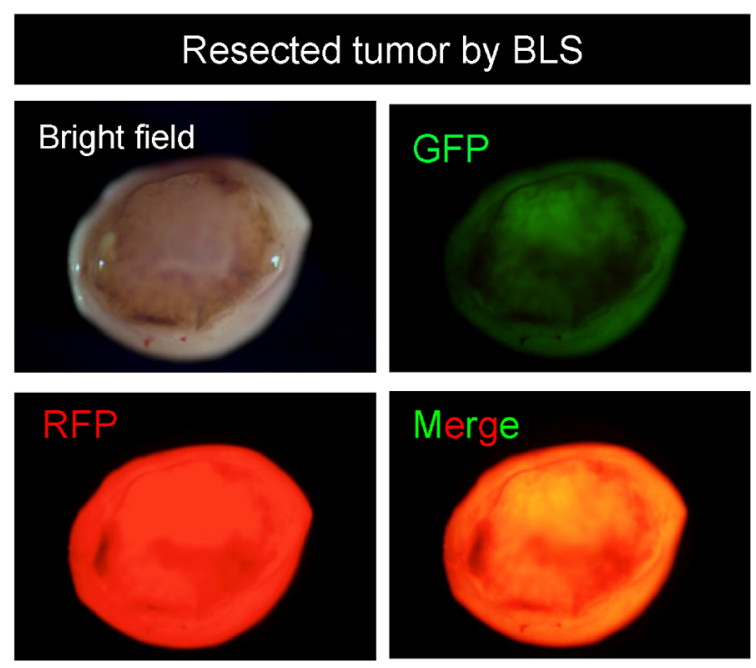

Resected tumor by OBP-401 FGS
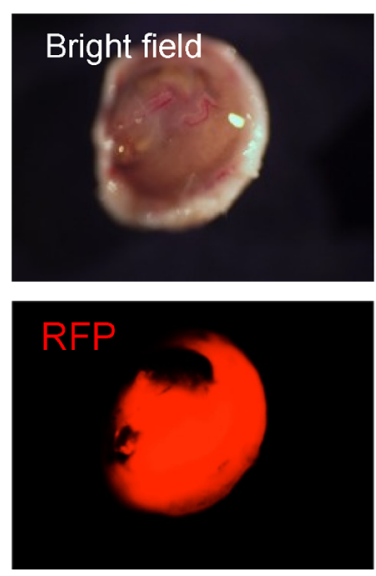

D

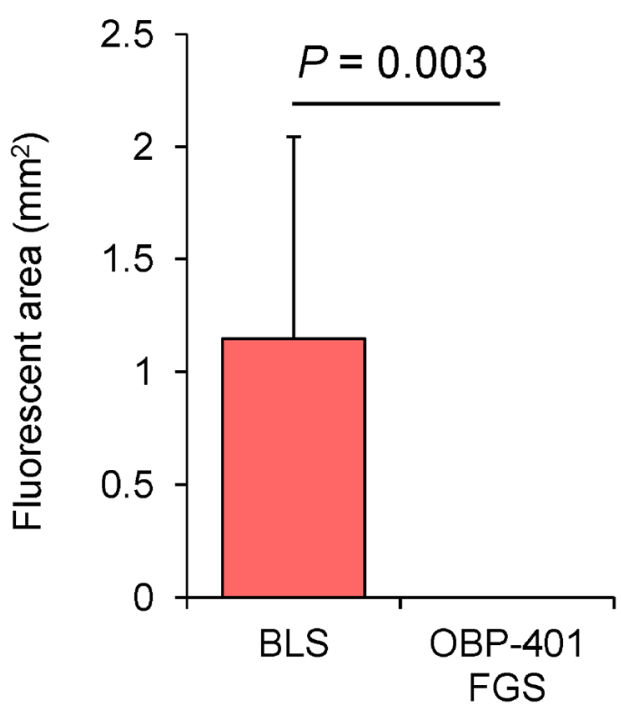

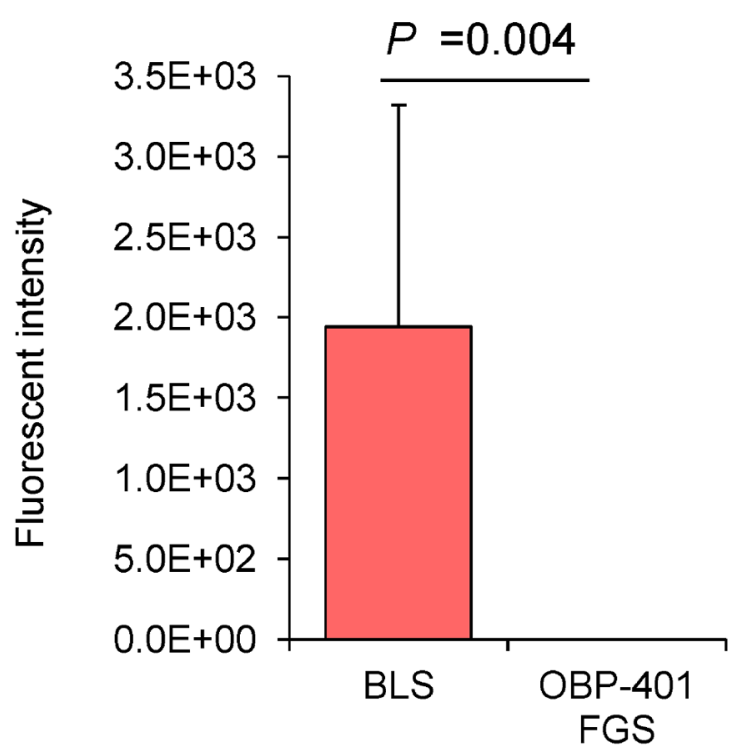

Figure 2 (Continued): C. Representative whole-tumor images of a tumor resected by BLS and a tumor resected by OBP-401 FGS using the OV100 whole body imaging system. D. Bar graph shows the comparison of fluorescent area in the surgical bed after BLS or OBP-401 FGS (left). Bar graph shows the comparison of fluorescence intensity in the surgical bed after BLS or OBP-401 FGS (right). Fluorescent area and fluorescence intensity are calculated with ImageJ software. Data are shown as average $\pm \mathrm{SD} . \mathrm{N}=12 . \mathrm{Scale}$ bars $=1 \mathrm{~cm}$.

was less than that by BLS or low-dose OBP-401 FGS due to the cytotoxic effects of OBP-401 (Figure 6E).

\section{OBP-401 based FGS resulted in recurrence-free surgery}

Fluorescence imaging showed that eight of twelve mice that underwent BLS had RFP-expressing local recurrences (Figure 7A, 7D and 7E). In contrast, there was no local recurrence in twelve mice which received lowdose OBP-401-FGS (Figure 7B, 7D and 7E). Moreover, there was also no local recurrence in ten mice which received minimal surgery after high-dose administration of OBP-401 (Figure 7C, 7D and 7E) (Table 1).
Labeling tumors with OBP-401 for FGS has none of the weaknesses of non-genetic labeling, particularly loss of label over time and limited expression of the marker used for labeling. OBP-401 may be a general method for labeling tumors that express telomerase, which are the vast majority, that could have broad application for FGS.

A Phase I clinical trial of i.t. injection of OBP301 , the parent of OBP-401, in patients with advanced solid tumors was well tolerated [12]. Melanoma occurs intracutaneously, and therefore OBP-401 could be directly delivered and be tested in the clinic in the near future.

Concepts and strategies of highly-selective tumor targeting that were previously developed [13-17] can take advantage of adenoviral targeting of tumors, including 
A
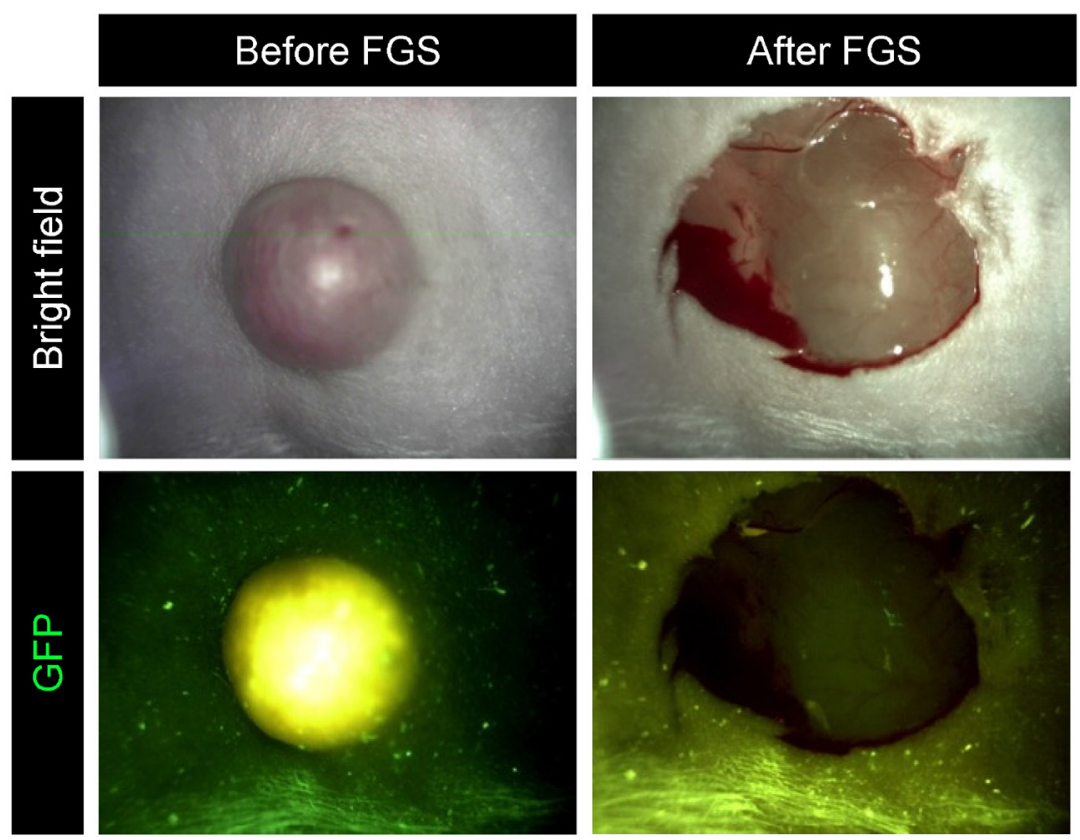

B
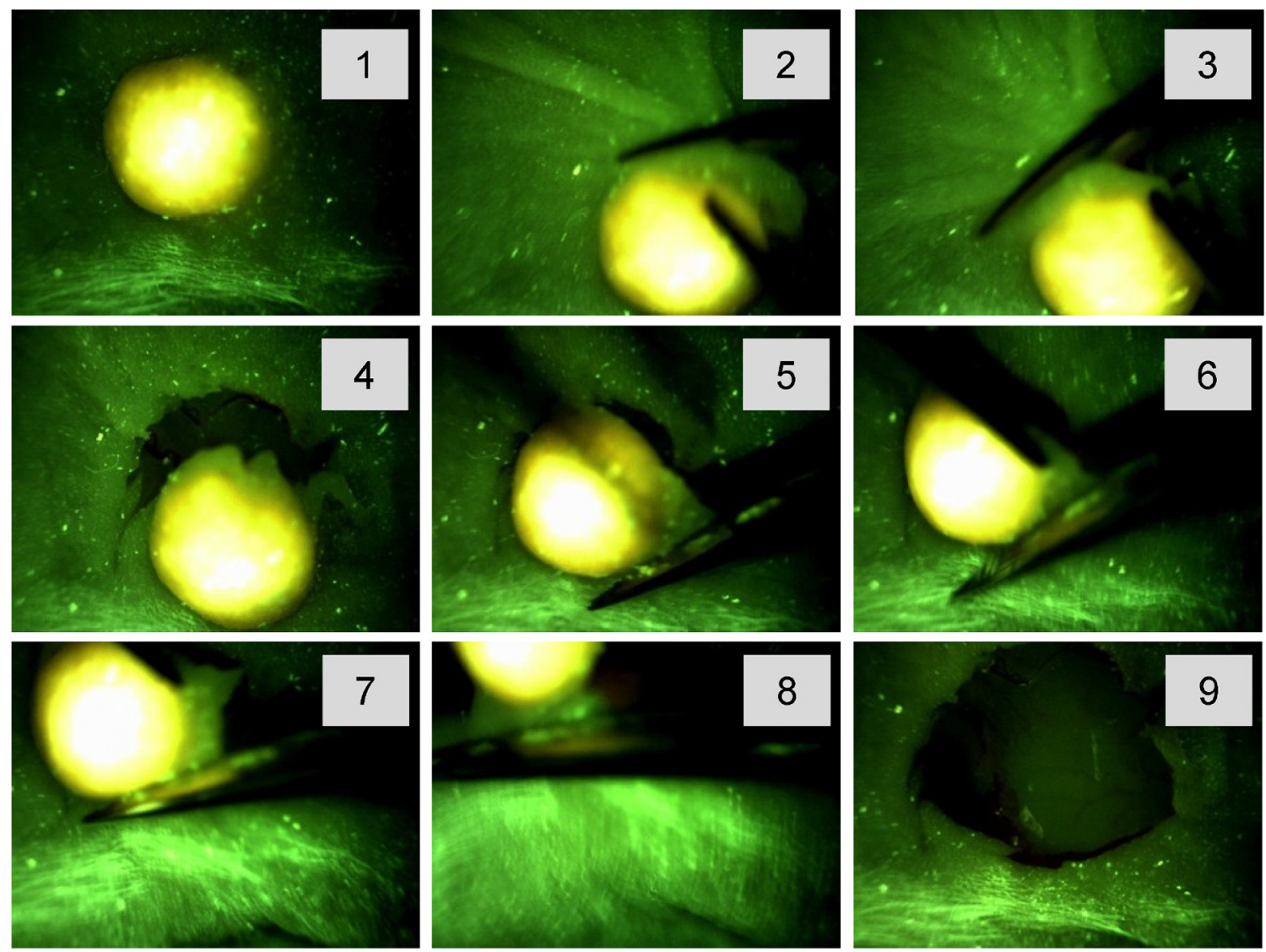

Figure 3. OBP-401 based FGS of melanoma using the Dino-Lite hand-held portable fluorescence scope. A. Representative whole tumor images of OBP-401-GFP labeled, RFP-expressing orthotopic malignant melanoma before and after OBP-401-FGS using the Dino-Lite hand-held fluorescence scope. B. Step-by-step procedure for OBP-401 based FGS of malignant melanoma using the Dino-Lite hand-held-fluorescence scope. 
A

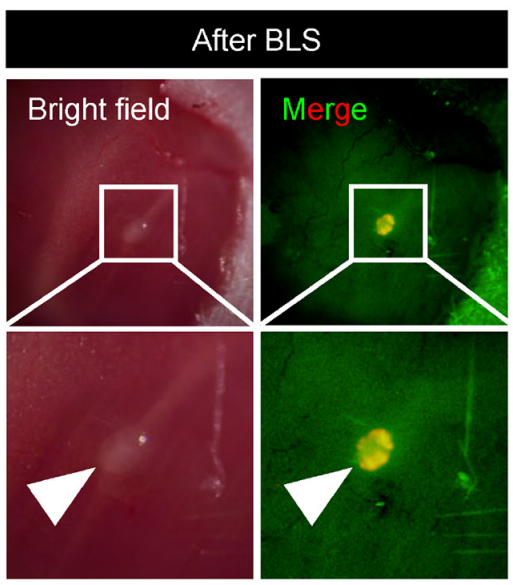

B

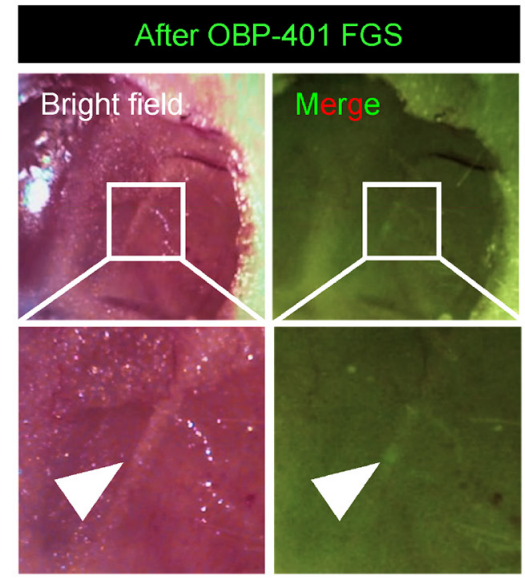

C
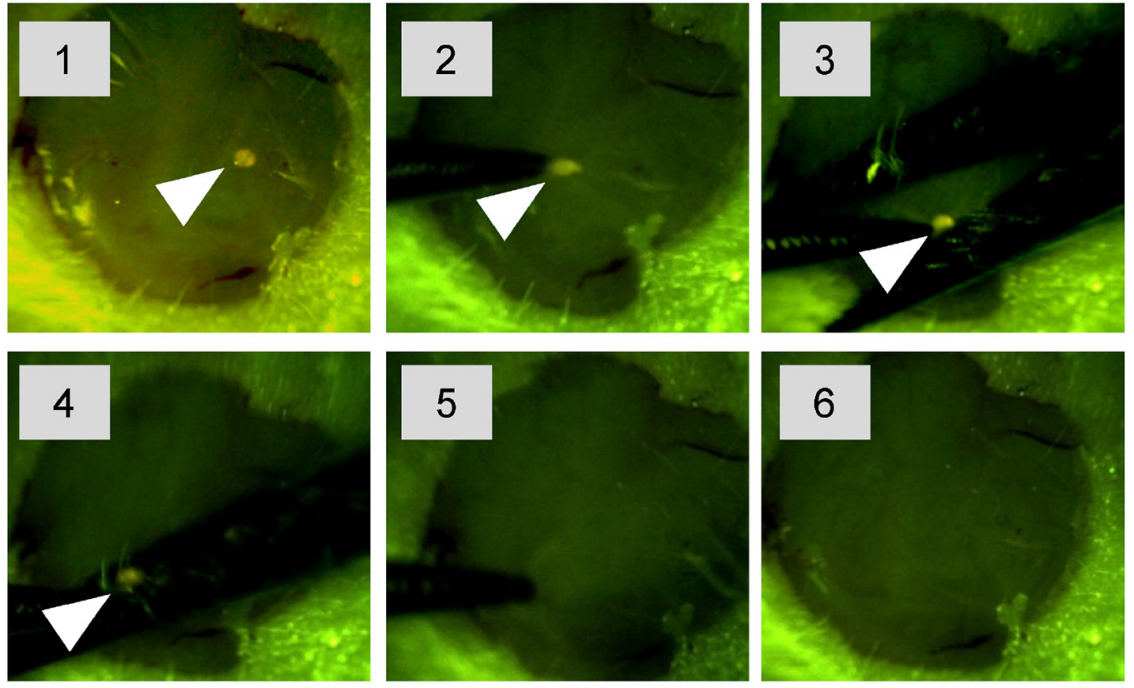

D

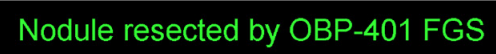

$E$
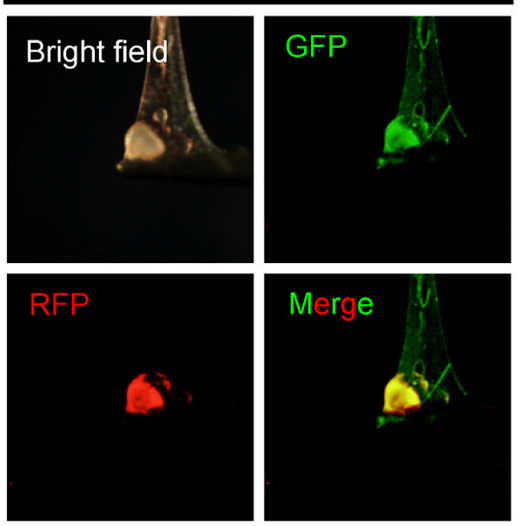

Nodule resected by OBP-401 FGS
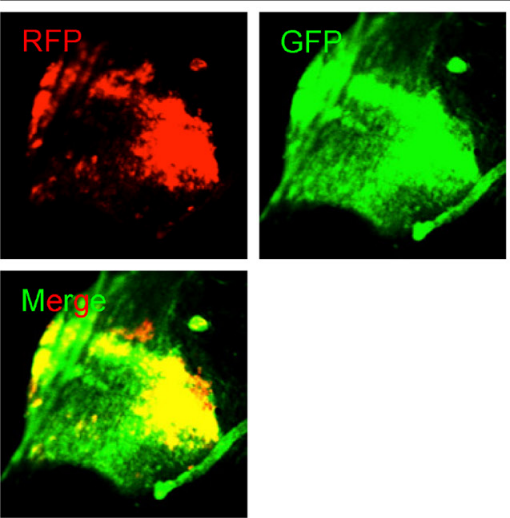

Figure 4. OBP-401 targeting visualizes residual malignant melanoma cells after BLS. A. Representative high-magnification images of the surgical bed of orthotopic malignant melanoma after BLS. B. OBP-401 targeting enabled visualization of residual tumor after BLS and results in complete resection. Representative high-magnification images of surgical bed after FGS of malignant melanoma. C. Step-by-step procedure of OBP-401 based FGS of residual malignant melanoma after BLS using the Dino-Lite hand-held fluorescence scope. D. Representative images of resected malignant melanoma nodule by FGS. Images were acquired with the OV100. E. Representative single-cell level images of resected malignant melanoma nodule after FGS. Images were acquired with the FV1000 confocal microscope. 
A
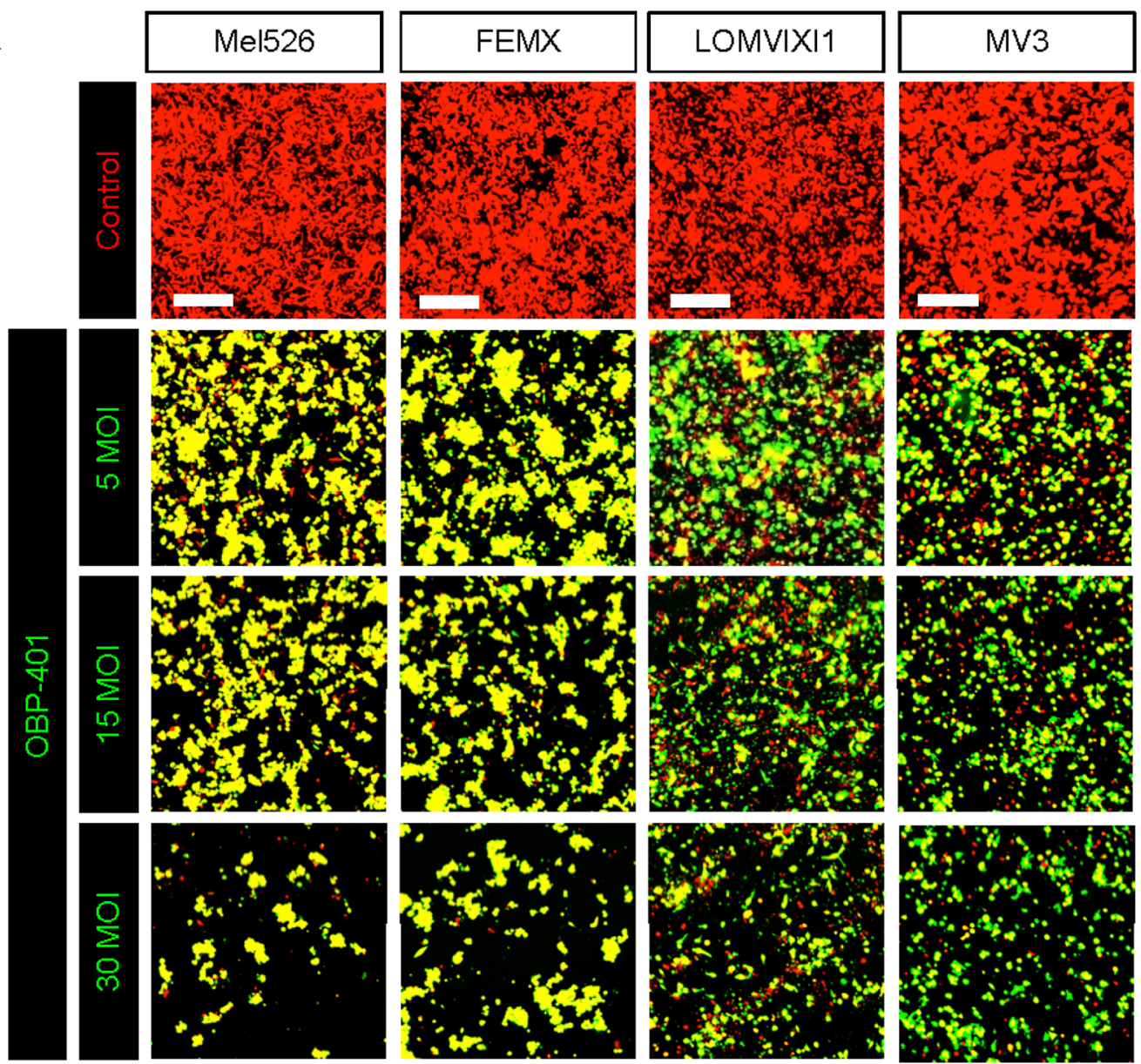

B
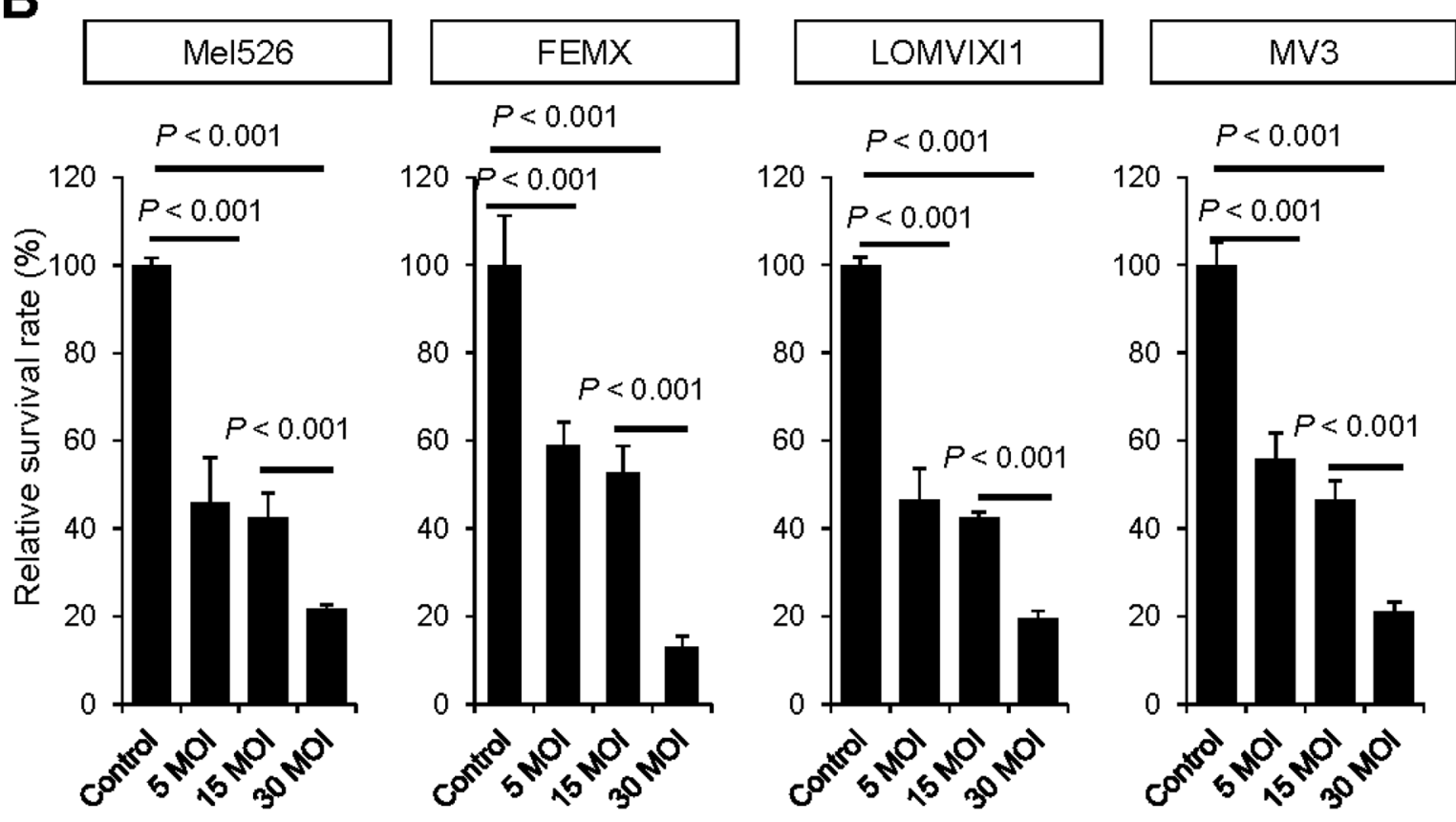

Figure 5. OBP-401 kills human malignant melanoma cell lines in vitro. A. Representative images of RFP-expressing human malignant melanoma cell lines, Mel526-RFP, FEMX1-RFP, LOMVIXI-RFP, and MV3-RFP, 7 days after targeting with OBP-401 at a MOI of 5, 15, and 30. B. Bar graphs show the surviving fraction of Mel526-RFP, FEMX1-RFP, LOMVIXI-RFP, and MV3-RFP cells 7 days after targeting with OBP-401 in vitro. The number of live cells was counted. Data are shown as average $\pm \mathrm{SD}$. $\mathrm{N}=5$. Scale bars $=250 \mu \mathrm{m}$. 
A
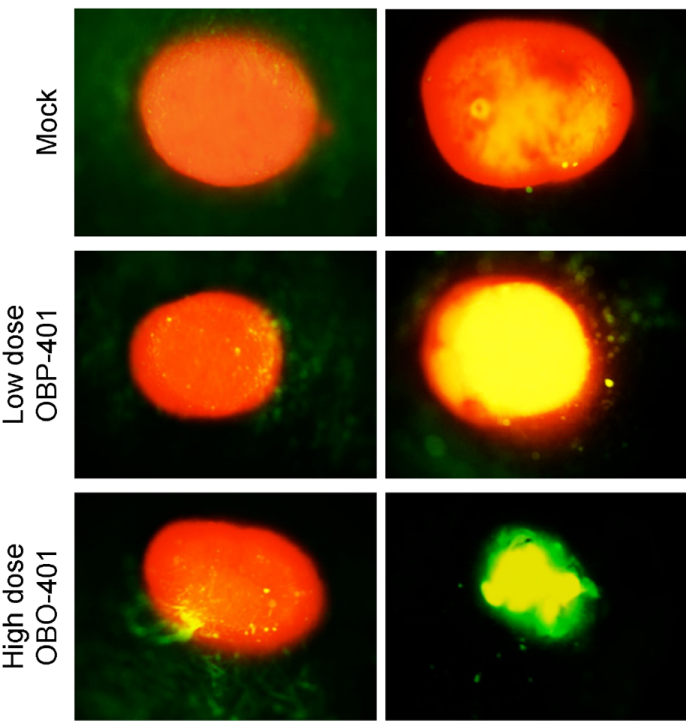

RFP/GFP/Merge
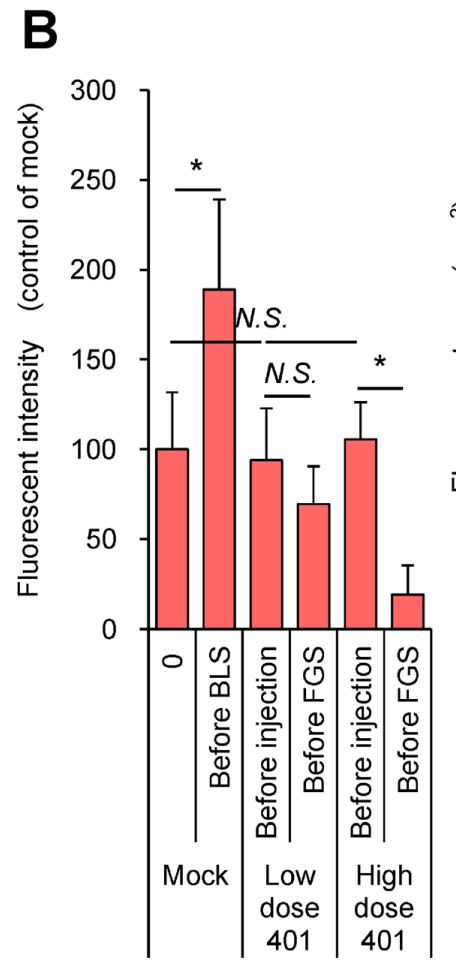

After injection
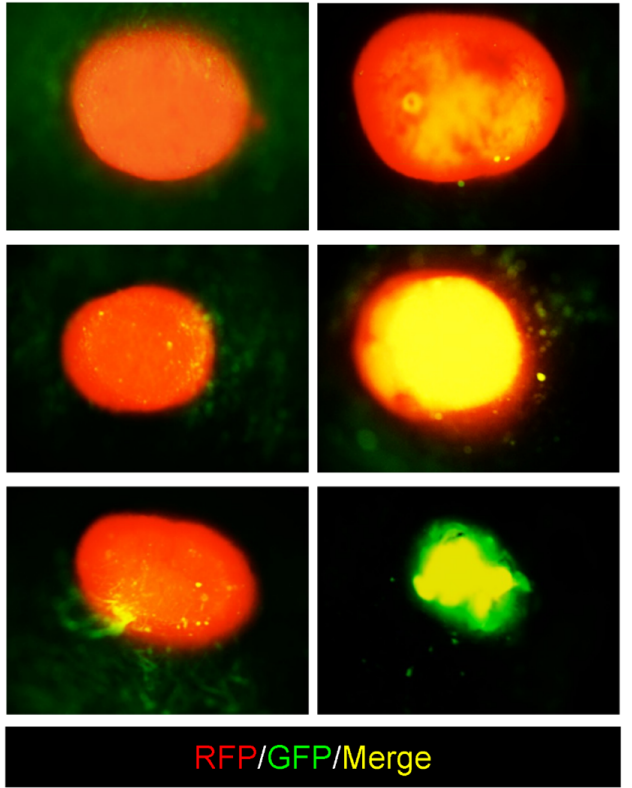

B

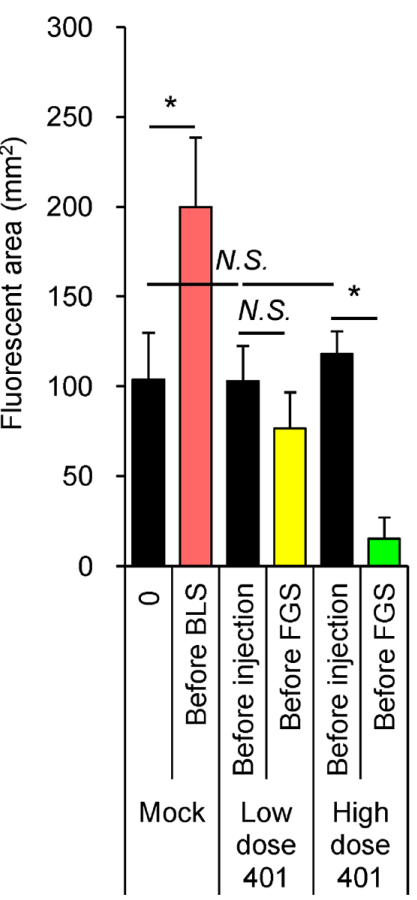

C
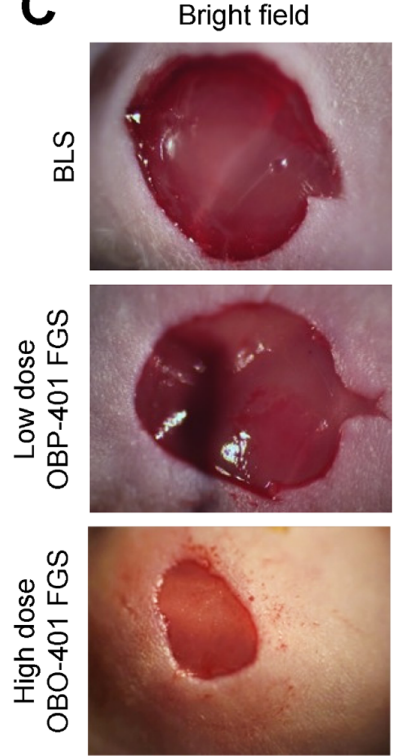

Fluorescence
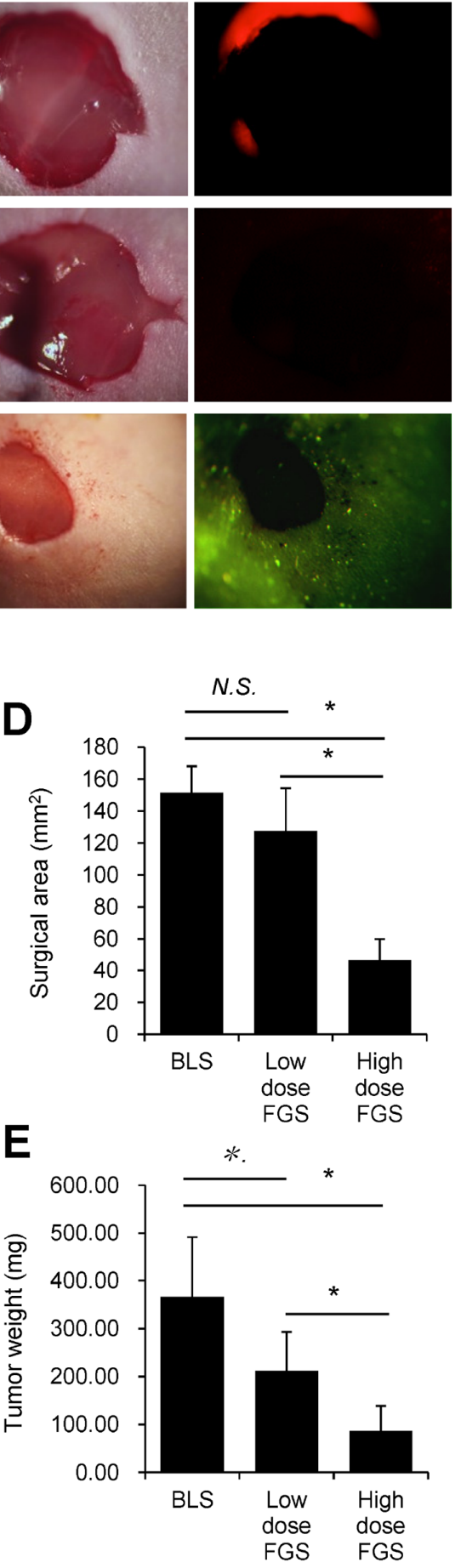

Figure 6. OBP-401 enables minimal precise surgery for orthotopic melanoma. A. Representative whole-tumor images of orthotopic malignant melanoma before and after mock-infection or before and after i.t. administration of low-dose OBP-401 $\left(1 \times 10^{8}\right.$ PFU) or high-dose OBP-401 (at $3 \times 10^{8}$ PFU). B. Bar graphs show the fluorescence intensity of a control tumor and low-dose or highdose OBP-401-targeted tumors (left). Fluorescent area is calculated with ImageJ software. Bar graphs show the fluorescence area of control tumor and low-dose or high-dose OBP-401-targeted tumors (right). Fluorescence intensity is calculated with ImageJ software. Data are shown as average $\pm \mathrm{SD} . \mathrm{N}=10$. C. Representative images of surgical area after BLS (upper) and low-dose (middle) or high-dose OBP-401-FGS (lower). Bright field images (left panels) and fluorescence images (right panels). D. Bar graphs show the comparison of surgical area after BLS, low-dose FGS, or high doe FGS. E. Bar graphs show the comparison of tumor weight after BLS, low-dose FGS, or high-dose FGS. Data are shown as average $\pm \mathrm{SD} . \mathrm{N}=10,{ }^{*} p<0.05$. 
A

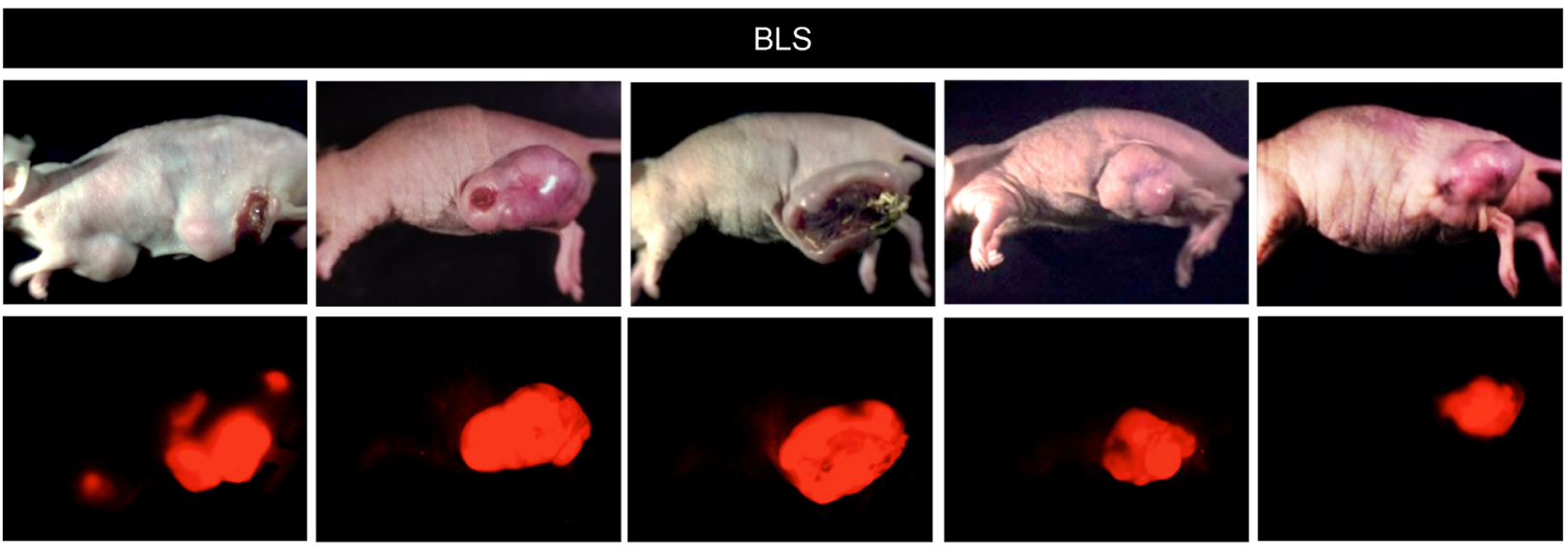

B

Low dose OBP-401 FGS
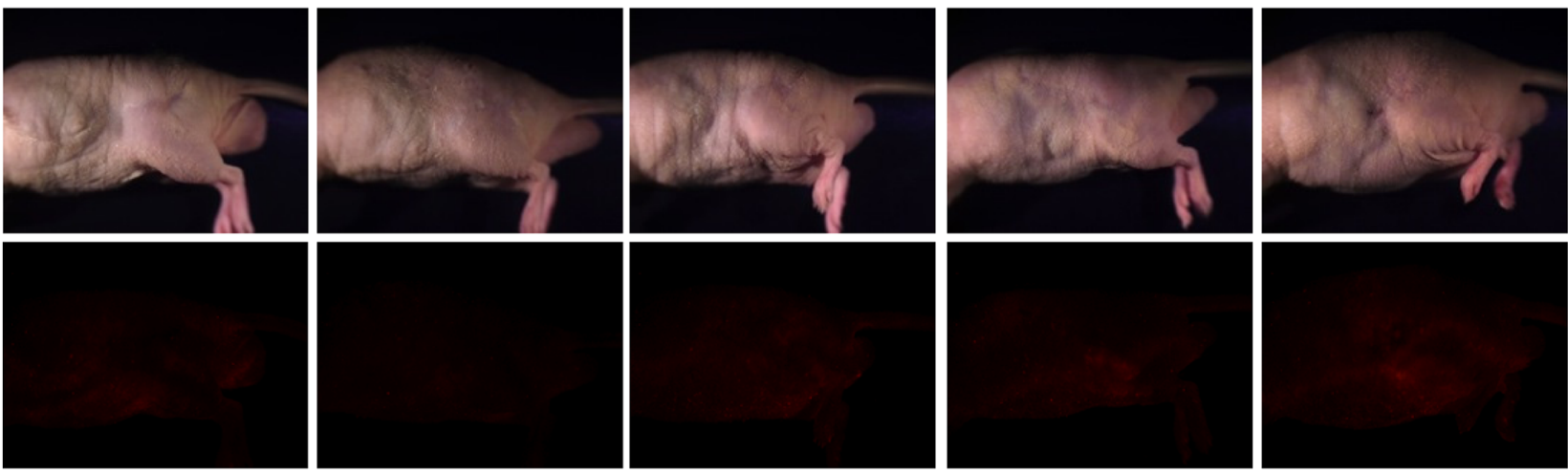

C

High dose OBP-401 FGS
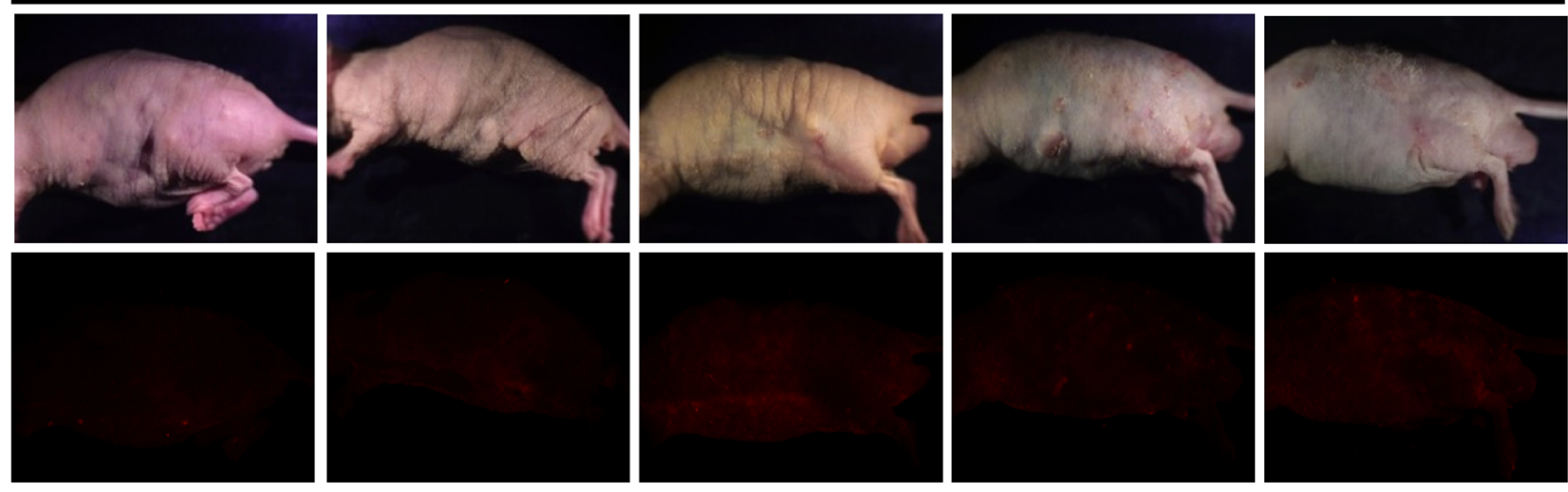

Figure 7. OBP-401-based FGS prevents local recurrence. A. Representative whole-body images of orthotopic malignant melanoma 90 days after BLS. B. Representative whole body images of orthotopic malignant melanoma 90 days after low-dose OBP401-FGS. C. Representative whole body images of orthotopic malignant melanoma 90 days after precise minimal FGS with high-dose administration of OBP-401. Upper panels in A, B, C: brightfield; lower panels: fluorescence.

(Continued) 
D
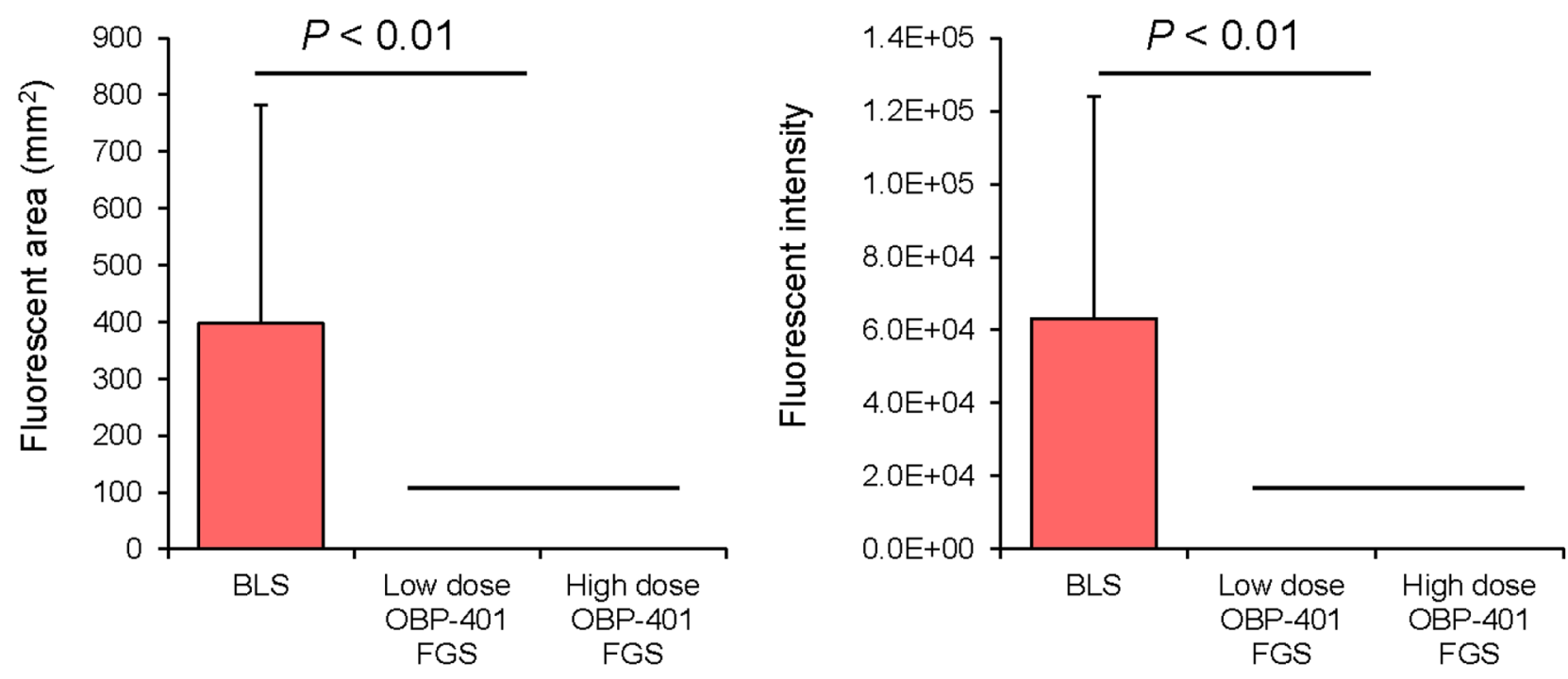

$\mathbf{E}$

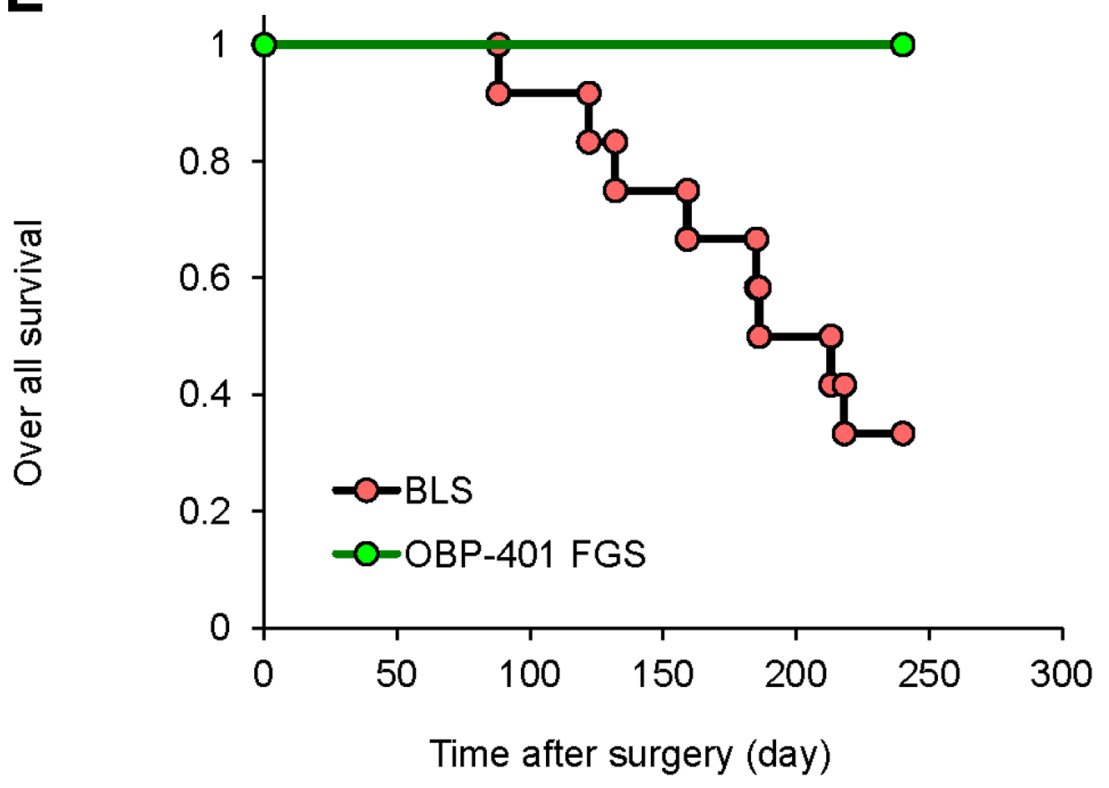

Figure 7 (Continued): D. Comparison of fluorescent areas of recurrent tumors after BLS, low-dose OBP-401-FGS, or high-dose OBP401-FGS (left panel). Fluorescent area is calculated with ImageJ software. Comparison of fluorescence intensity of recurrent tumors after BLS, low-dose OBP-401-FGS, or high-dose OBP-401-FGS (right panel). Fluorescence intensity is calculated with ImageJ software. Data are shown as average $\pm \mathrm{SD}$. $\mathrm{N}=10$. E. Kaplan-Meyer curves show the over-all survival after BLS or OBP-401-FGS.

tissue-selective therapy which focuses on unique properties of normal and tumor tissues $[13,15,18]$. OBP401 can possibly overcome de-differentiation of a tumor leading to resistance to targeted chemotherapy, because the targeted protein or pathway may no longer be expressed [18], since OBP-401 does not depend on such targets [15], but depends only on telomerase expression which is expected to be stable in cancer cells. OBP-401 may also be effectively combined with teratogens which could selectively effect cancer cells that are dedifferentiated [14].
Since OBP-401 can decoy quiescent cancer cells to begin to cycle [19], OBP-401 could be effectively combined with agents which selectively target proliferating cancer cells [16], where normal cells are protected by agents which induce wild type p53 [17].

Other fluorescence methods of labeling tumors are also available. Fluorescence labeling at long wavelengths, such as near infrared (NIR), enables potential greater depth of visualization of the label, but such non-genetic methods will not label recurrent tumors [20]. 
Table 1. Tumor recurrence.

\begin{tabular}{|l|c|c|}
\hline \multicolumn{1}{l}{ Local recurrence } & Positive & Negative \\
\hline Conventional BLS & 8 & 4 \\
\hline Low-dose OBP-401 FGS & 0 & 12 \\
\hline High-dose OBP-401 FGS & 0 & 10 \\
\hline
\end{tabular}

$* P=0.001, * * P=0.003$

\section{MATERIALS AND METHODS}

\section{GFP-expressing telomerase-specific adenovirus}

In OBP-401, the promoter element of the human telomerase reverse transcriptase ( $h T E R T$ ) gene drives the expression of E1A and E1B genes, linked to an internal ribosome entry site, for selective replication only in cancer cells. The GFP gene is driven by the CMV promoter inserted in OBP-401 [21].

\section{Cell line and cell culture}

Red fluorescent protein-expressing human malignant melanoma cell lines Mel526-RFP, FEMXRFP, LOMVIXI-RFP, and MV3-RFP (AntiCancer, Inc., San Diego, CA) were maintained and cultured in DMEM medium with $10 \%$ fetal bovine serum (FBS) and 5\% penicillin/streptomycin.

\section{In vitro imaging}

Time-course imaging of OBP-401-GFP labeling of melanoma cell lines was performed with an FV1000 confocal laser-scanning microscope (Olympus, Tokyo, Japan).

\section{Animal experiments}

Athymic $(n u / n u)$ nude mice (AntiCancer, Inc., San Diego, CA) were kept in a barrier facility under HEPA filtration. Mice were fed with autoclaved laboratory rodent diet (Tecklad LM-485, Western Research Products). All animal studies were conducted in accordance with the principles and procedures outlined in the National Institutes of Health Guide for the Care and Use of Laboratory Animals under Assurance Number A3873- 01.

\section{Orthotopic melanoma model}

RFP-expressing FEMX1 cells $\left(5 \times 10^{6}\right)$ suspended in Matrigel $(20 \mathrm{uL})$ were inoculated into the left femoral skin of athymic nude mice (5-weeks old). Tumor progression was monitored by noninvasive fluorescence imaging (OV100) [22].

\section{OBP-401 based fluorescence-guided surgery (OBP-401-FGS)}

All animal procedures were performed under anesthesia using s.c. administration of a ketamine mixture [10 $\mu$ l ketamine $\mathrm{HCl}, 7.6 \mu$ xylazine, $2.4 \mu \mathrm{l}$ acepromazine maleate, and $10 \mu \mathrm{l}$ PBS]. Orthotopic melanoma labeled with GFP by OBP-401 was observed with noninvasive fluorescence imaging using the OV100 Small Animal Imaging System (Olympus) [22]. OBP-401 FGS was performed using either the OV100 or a Dino-Lite hand-held fluorescence scope (Dino-Lite digital camera, AM4113TGFBW Dino-Lite Premier; AnMo Electronics Corp., Hsinchu, Taiwan) [11]. After surgery, the presence of cancer cells was observed with the OV100 and Dino-Lite. If there were residual cancer cells, an additional resection was performed [7].

\section{Statistical analysis}

Data are shown as means $\pm \mathrm{SD}$. For comparison between two groups, significant differences were determined using the Student's $t$-test. For comparison of more than two groups, statistical significance was determined with a one-way analysis of variance (ANOVA) followed by a Bonferroni multiple group comparison test. Pearson chi-square analysis was used to compare the rate of local recurrence between BLS and OBP-401-FGS. Statistical analysis for disease-free survival and over-all survival was performed using the Kaplan-Meier test along with log-rank test. Pearson chi-square analysis was used to evaluate the rate of local recurrence and lung metastasis between BLS and OBP-401-FGS. $P$ values of $<0.05$ were considered significant.

\section{Author's contributions}

Conception and design:SY, TF, RMH

Development of methodology: SY, KT, HK, RMH

Acquisition of data (provided animals, provided facilities, etc): RMH, YU, HK, SY

Analysis and interpretation of data (e.g., statistical analysis, biostatistics, computational analysis): SY, KT, SK, RMH SY, RMH 
Administrative, technical, or material support:

RMH, HK, TF, YU

Study supervision: $\mathrm{RMH}, \mathrm{HK}, \mathrm{SK}, \mathrm{MB}, \mathrm{TF}$

\section{DEDICATION}

This paper is dedicated to the memory of A.R. Moossa, M.D.

\section{GRANT SUPPORT}

This study was supported by grants-in-Aid from the Ministry of Education, Science and culture, Japan and grants from the Ministry of Health and Welfare, Japan. This study was also supported in part by National Cancer Institute grants CA 132971 and CA142669.

\section{CONFLICTS OF INTEREST}

Y. Urata is President \& CEO of Oncolys BioPharma, Inc., the manufacturer of OBP-401. T. Fujiwara is a consultant of Oncolys BioPharma, Inc. S. Yano, K. Takehara, and R. M. Hoffman are or were unsalaried associates at AntiCancer, Inc.

\section{REFERENCES}

1. Slominski AT, Carlson AJ. Melanoma resistance: bright future for academicians and challenge for patient advocates. Mayo Clin Proc 2014; 89:429-433.

2. Hoffman RM. The multiple uses of fluorescent proteins to visualize cancer in vivo. Nature Reviews Cancer 2005; 5:796-806.

3. Hoffman RM, Yang M. Subcellular imaging in the live mouse. Nature Protocols 2006; 1:775-782.

4. Kishimoto H, Zhao M, Hayashi K, Urata Y, Tanaka N, Fujiwara T, Penman S, Hoffman RM. In vivo internal tumor illumination by telomerase-dependent adenoviral GFP for precise surgical navigation. Proc Natl Acad Sci USA. 2009; 106:14514-14517.

5. Kishimoto H, Aki R, Urata Y, Bouvet M, Momiyama M, Tanaka N, Fujiwara T, Hoffman RM. Tumor-selective adenoviral-mediated GFP genetic labeling of human cancer in the live mouse reports future recurrence after resection. Cell Cycle 2011; 10:2737-2741.

6. Yano S, Miwa S, Kishimoto H, Toneri M, Hiroshima Y, Yamamoto M, Bouvet M, Urata Y, Tazawa H, Kagawa S, Funiwara T, Hoffman RM. Experimental curative fluorescence-guided surgery of highly invasive glioblastoma multiforme selectively labeled with a killer-reporter adenovirus. Molecular Therapy 2015; 23:1182-1188.

7. Yano S, Miwa S, Kishimoto H, Uehara F, Tazawa H, Toneri M, Hiroshima Y, Yamamoto M, Urata Y, Kagawa S, Bouvet M, Funiwara T, Hoffman RM. Targeting tumors with a killer-reporter adenovirus for curative fluorescence-guided surgery of soft-tissue sarcoma. Oncotarget 2015; 6:1313313148. doi: 10.18632/oncotarget.3811.

8. Yano S, Hiroshima Y, Maawy A, Kishimoto H, Suetsugu A, Miwa S, Toneri M, Yamamoto M, Katz MHG, Fleming JB, Urata Y, Tazawa H, Kagawa S, Bouvet M, Fujiwara T, Hoffman RM. Color-coding cancer and stromal cells with genetic reporters in a patient-derived orthotopic xenograft (PDOX) model of pancreatic cancer enhances fluorescenceguided surgery. Cancer Gene Therapy 2015; 22:344-350.

9. Yano S, Zhang Y, Miwa S, Kishimoto H, Urata Y, Bouvet M, Kagawa S, Fujiwara T, Hoffman RM. Precise navigation surgery of tumors in the lung in mouse models enabled by in situ fluorescence labeling with a killer-reporter adenovirus. BMJ Open Respiratory Research 2015; 2:e000096.

10. Yano S, Miwa S, Urata Y, Tazawa H, Kagawa S, Bouvet M, and Hoffman RM. Eradication of osteosarcoma by fluorescence-guided surgery with tumor labeling by a killer-reporter adenovirus. J. Orthopaedic Res. 2015. doi: 10.1002/jor.23073.

11. Hiroshima Y, Maawy A, Sato S, Murakami T, Uehara F, Miwa S, Yano S, Momiyama M, Chishima T, Tanaka K, Bouvet M, Endo I, Hoffman RM. Hand-held highresolution fluorescence imaging system for fluorescenceguided surgery of patient and cell-line pancreatic tumors growing orthotopically in nude mice. J Surg Res. 2014; 187:510-517.

12. Nemunaitis J, Tong AW, Nemunaiti M, Senzer N, Phadke AP, Bedell C, Adams N, Zhang YA, Maples PB, Chen S, Pappen B, Burke J, Ichimaru D, Urata Y, Fujiwara T. A phase I study of telomerase-specific replication competent oncolytic adenovirus (telomelysin) for various solid tumors. Mol Ther 2010; 18:429-434.

13. Blagosklonny MV. Matching targets for selective cancer therapy. Drug Discov Today 2003; 8:1104-1107.

14. Blagosklonny MV. Teratogens as anti-cancer drugs. Cell Cycle 2005; 4:1518-1521.

15. Blagosklonny MV. Treatment with inhibitors of caspases, that are substrates of drug transporters, selectively permits chemotherapy-induced apoptosis in multidrug-resistant cells but protects normal cells. Leukemia 2001; 15:936-941.

16. Blagosklonny MV. Target for cancer therapy: proliferating cells or stem cells. Leukemia 2006; 20:385-391.

17. Apontes $\mathrm{P}$, Leontieva $\mathrm{OV}$, Demidenko $\mathrm{ZN}$, Li F, Blagosklonny MV. Exploring long-term protection of normal human fibroblasts and epithelial cells from chemotherapy in cell culture. Oncotarget 2011; 2:222-233. doi: 10.18632/ oncotarget.248.

18. Blagosklonny MV. Tissue-selective therapy of cancer. Br J Cancer 2003;89:1147-1151.

19. Yano S, Tazawa H, Hashimoto Y, Shirakawa Y, Kuroda S, Nishizaki M, Kishimoto H, Uno F, Nagasaka T, Urata Y, Kagawa S, Hoffman RM, Fujiwara T. A genetically engineered oncolytic adenovirus decoys and lethally traps quiescent cancer stem-like cells into $\mathrm{S} / \mathrm{G}_{2} / \mathrm{M}$ phases. Clin. Cancer Res. 2013; 19:6495-6505. 
20. Kobayashi H, Ogawa M, Alford R, Choyke PL, Urano Y. New strategies for fluorescent probe design in medical diagnostic imaging. Chemical Rev 2010; 110:2620-2640.

21. Kishimoto H, Kojima T, Watanabe Y, Kagawa S, Fujiwara T, Uno F, Teraishi F, Kyo S, Mizuguchi H, Hashimoto Y, Urata Y, Tanaka N, Fujiwara T. In vivo imaging of lymph node metastasis with telomerase-specific replication-selective adenovirus. Nat Med 2006; 12:1213-1219.
22. Yamauchi K, Yang M, Jiang $\mathrm{P}, \mathrm{Xu} \mathrm{M}$, Yamamoto N, Tsuchiya H, Tomita K, Moossa AR, Bouvet M, Hoffman RM. Development of real-time subcellular dynamic multicolor imaging of cancer-cell trafficking in live mice with a variable-magnification whole-mouse imaging system. Cancer Res. 2006; 66:4208-4214. 